\title{
Increased AST/ALT ratio in azotaemic dogs infected with Babesia canis
}

\author{
W. Zygner1 ${ }^{1}$, O. Gójska-Zygner², L.J. Norbury ${ }^{3}$ H. Wędrychowicz ${ }^{1,3}$ \\ ${ }^{1}$ Division of Parasitology and Parasitic Diseases, Department of Preclinical Sciences, \\ Faculty of Veterinary Medicine, Warsaw University of Life Sciences - SGGW, \\ Ciszewskiego 8, 02-786 Warsaw, Poland \\ ${ }^{2}$ Center of Small Animal Health Clinic Multiwet, Gagarina 5, 00-753 Warsaw, Poland \\ ${ }^{3}$ W. Stefański Institute of Parasitology, Twarda 51/55, 00-818 Warsaw, Poland
}

\begin{abstract}
The AST/ALT ratio was estimated in 182 dogs infected with Babesia canis. Among these dogs 65 had anaemia and 68 were azotaemic. Student's $t$ test was used to compare means of the AST/ALT ratio in anaemic and non-anaemic dogs, and in azotaemic and non-azotaemic dogs $(p<0.05)$. The differences in AST/ALT ratio between anaemic $(1.52 \pm 1.15)$ and non-anaemic $(1.76 \pm 1.34)$ dogs were statistically insignificant $(p=0.23)$, however, the comparison of AST/ALT ratio between azotaemic $(2.68 \pm 1.52)$ and non-azotaemic $(1.08 \pm 0.53)$ dogs revealed a significantly higher value of this index in azotaemic dogs $(\mathrm{p}=0.00)$. The present results suggest that kidney injury contributed to increased AST activity in these dogs.
\end{abstract}

Key words: AST/ALT ratio, azotaemia, Babesia canis, canine babesiosis, kidney injury, dogs

\section{Introduction}

Babesia canis (formerly known as $B$. canis canis) is a tick-transmitted protozoan parasite of dogs. Infection with this parasite leads to development of the systemic disease canine babesiosis. The literature describes at least 5 species of the genus Babesia that infect dogs (Kjemtrup et al. 2006, Irwin 2009). Yet, to date, in Poland only one species of this genus has been detected in dogs and ticks, i.e. B. canis (Adaszek and Winiarczyk 2008, Zygner et al. 2008, Welc-Falęciak et al. 2009, Zygner et al. 2009).

Anaemia, azotaemia and increased liver enzyme activities were described in dogs infected with $B$. canis in Poland (Zygner et al. 2007a,b, Abramowicz 2008). Hypoxia is considered as one of the causes of the liver and kidney injury during the course of canine babesiosis (Mhthe et al. 2007, Zygner et al. 2011). A previous study showed that anaemia had no influence on liver injury (Zygner et al. 2011). However, in that study, and also in other works on canine babesiosis, the level of aspartate aminotransferase (AST) activity was found to be higher than that of alanine aminotransferase (ALT) activity in a larger number of serum samples, which suggested that increased AST activity resulted not only from hepatopathy (Furlanello et al. 2005, Zygner et al. 2007b). We hypothesised that this finding might be a consequence of kidney injury or haemolysis, with kidney injury seemingly the more probable explanation for increased AST activity (Zygner et al. 2011). Therefore, we supposed that the AST/ALT ratio (de Ritis

Correspondence to: W. Zygner, e-mail: wojciechzygner@yahoo.pl 
Table 1. Number of samples in 4 groups after division of all 182 serum and blood samples from dogs infected with $B$. canis.

\begin{tabular}{cccc}
\hline Division into groups & Group A & Group B & Total (group A + B) \\
\hline Group C & 22 & 46 & 68 \\
Group D & 43 & 71 & 114 \\
Total (group C + D) & 65 & 117 & 182 \\
\hline
\end{tabular}

Group A, samples from anaemic dogs; Group B, samples from non-anaemic dogs; Group C, samples from azotaemic dogs; Group D, samples from non-azotaemic dogs.

quotient) might increase in azotaemic dogs during babesiosis and subsequently that the AST/ALT ratio might be another useful indicator of azotaemia in dogs infected with $B$. canis. The present study is a continuation of previous research on liver enzyme activity during the course of canine babesiosis (Zygner et al. 2011).

In this study, the influence of anaemia and azotaemia on the AST/ALT ratio during canine babesiosis was investigated, with the purpose of determining whether the more frequently observed increased AST activity than ALT activity (leading to an increase in de Ritis quotient) was connected with anaemia (presumably resulting from haemolysis) or with azotaemia (presumably resulting from renal injury).

\section{Materials and Methods}

In a previous study, whole blood and serum was collected from 230 dogs infected with Babesia canis prior to the treatment (Zygner et al. 2011). Liver enzyme activities (ALT, AST and ALP) were determined by a clinical chemistry analyzer (XL 640, Erba Mannheim). Concurrently, using the same chemistry analyzer, serum urea and creatinine concentrations were determined. Erythrocyte parameters, such as red blood cell count (RBC), haematocrit ( $\mathrm{Ht})$, haemoglobin concentration $(\mathrm{Hb})$, mean corpuscular haemoglobin concentration (MCHC), and mean corpuscular volume (MCV) were assessed with an automatic haematologic analyzer (Diatron, Abacus).

The obtained results allowed to divide samples into two groups: group A (samples from anaemic dogs) and group B (samples from non-anaemic dogs). Concurrently, all serum and blood samples were also divided into other two groups: group C (samples from azotaemic dogs) and group D (samples from non-azotaemic dogs). However, 48 samples were excluded from the study owing to an inability to clearly identify azotaemia (i.e. increased concentration of serum urea and creatinine concentration within reference intervals in the same sample). Thus, 182 serum and blood samples collected in the previous research were included in this study. Division of all 182 samples into groups is presented in Table 1. The number of serum and blood samples in groups were as follows: 65 samples in group A, 117 samples in group B, 68 samples in group C, and 114 samples in group D. The results were analysed using the Statistica 8.0 program. Student's $t$ test was used to compare the means of the AST/ALT ratio between group A and B, and between group $\mathrm{C}$ and $\mathrm{D}$. A value at $p<0.05$ was considered as significant. Correlations between the AST/ALT ratio and azotaemic parameters (i.e. serum urea and creatinine) were calculated.

\section{Results}

Anaemia was detected in $35.7 \%(65 / 182 ; 95 \%$ CI: $28.7-42.7)$ of dogs included in this study. Means and standard deviations $(\bar{x} \pm \mathrm{SD})$ of $\mathrm{RBC}, \mathrm{Ht}$, and $\mathrm{Hb}$ in this group (group A) were $4.22 \pm 0.99 \times 10^{12} / \mathrm{L}, 0.28$ $\pm 0.06 \mathrm{~L} / \mathrm{L}$ and $5.97 \pm 1.33 \mathrm{mmol} / \mathrm{L}$, respectively. The remaining dogs (117/182; 64.3\%; 95\% CI: 57.3 - 71.3) had no anaemia. In this group of the animals (group B) means and standard deviations $(\bar{x} \pm \mathrm{SD})$ of RBC, $\mathrm{Ht}$, and $\mathrm{Hb}$ were within reference intervals (RBC, 5.5-8.0 × 10 $12 / \mathrm{L} ; \mathrm{Ht}, 0.37-0.55 \mathrm{~L} / \mathrm{L}, \mathrm{Hb}, 7.45-11.17$ $\mathrm{mmol} / \mathrm{L})$ and amounted to $6.45 \pm 0.67 \times 10^{12} / \mathrm{L}, 0.43$ $\pm 0.04 \mathrm{~L} / \mathrm{L}$ and $9.20 \pm 0.96 \mathrm{mmol} / \mathrm{L}$, respectively. Azotaemia was detected in $37.4 \%$ (68/182; $95 \%$ CI: $30.3-44.5)$ of dogs included in this study. Means and standard deviations $(\bar{x} \pm \mathrm{SD})$ of serum urea and creatinine concentrations in this group (group C) amounted to $178.84 \pm 95.55 \mathrm{mg} / \mathrm{dL}$ and $3.25 \pm 2.03$ $\mathrm{mg} / \mathrm{dL}$, respectively. The remaining dogs (114/182; $62.6 \%$; 95\% CI: 55.5 - 69.7) had no azotaemia. In this group (group D) means and standard deviations $(\bar{x}$ \pm SD) of serum urea and creatinine concentrations were within reference intervals (urea, $2045 \mathrm{mg} / \mathrm{dL}$; creatinine, $1.01 .7 \mathrm{mg} / \mathrm{dL}$ ) and amounted to 34.45 $\pm 8.2803 \mathrm{mg} / \mathrm{dL}$ and $1.00 \pm 0.2103 \mathrm{mg} / \mathrm{dL}$, respectively.

AST activity above reference intervals (1-37 UI/L) was observed in 169 (92.9\%; 95\% CI: 89.1 - 96.6) out of 182 samples. Increased ALT activity above reference intervals (3-50 UI/L) was observed in 119 (65.4\%; 95\% CI: 58.4 - 72.4) out of 182 samples. 


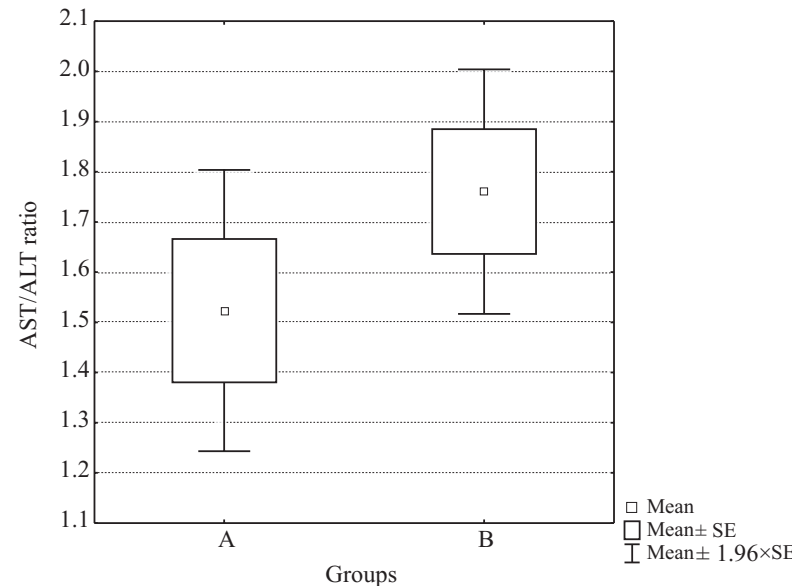

Fig. 1. Comparison of AST/ALT ratios in anaemic (group A) and non-anaemic dogs (group B) infected with B. canis. Student's $t$ test result: $t=-1.20, p=0.23$ (SE, standard error; $t$, a value of $t ; p$, a value of $p$ ).

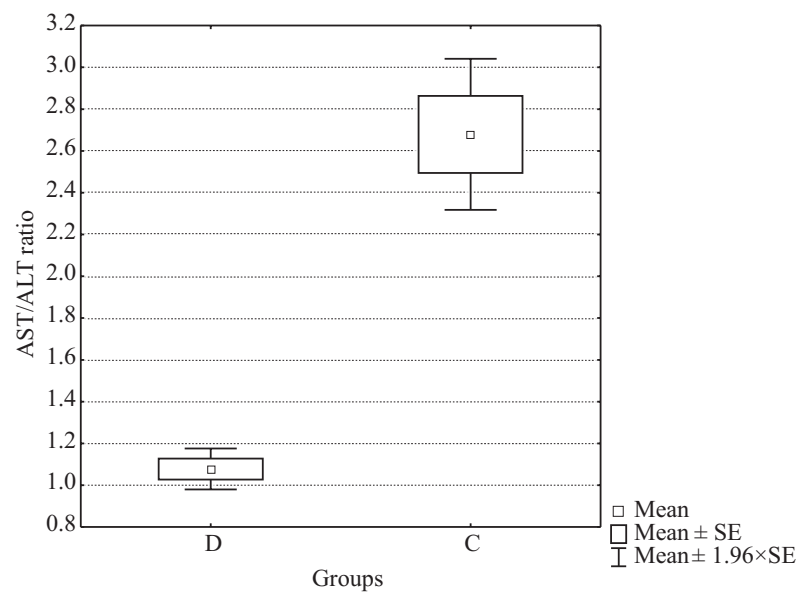

Fig. 2. Comparison of the AST/ALT ratio in azotaemic (group C) and non-azotaemic dogs (group D) infected with B. canis. Student's $t$ test result: $t=-10.25, p=0.00$ (SE, standard error; $t$, a value of $t ; p$, a value of $p$ ).

Mean AST and ALT activities $( \pm$ SD) in all 182 examined samples were $116.92 \pm 91.85 \mathrm{UI} / \mathrm{L}$ and 89.84 $\pm 89.94 \mathrm{UI} / \mathrm{L}$, respectively. The mean AST/ALT ratio $( \pm \mathrm{SD})$ of all 182 examined samples amounted to $1.68 \pm 1.28$.

Mean AST/ALT ratios $( \pm \mathrm{SD})$ in groups $\mathrm{A}$ and $\mathrm{B}$ amounted to $1.52 \pm 1.15$ (group A) and $1.76 \pm 1.34$ (group B). Student's $t$ test did not reveal statistically significant differences between means of the AST/ALT ratios in groups A and B (Fig. 1). Mean AST/ALT ratios $( \pm \mathrm{SD})$ in groups $\mathrm{C}$ and $\mathrm{D}$ amounted to $2.68 \pm 1.52$ (group C) and $1.08 \pm 0.53$ (group D). Comparison of AST/ALT ratio between groups C and $\mathrm{D}$ using Student's $t$ test revealed statistically significant difference (Fig. 2). High correlations between AST/ALT ratio and serum urea concentration $(r=0.68, p=0.00)$, and between AST/ALT ratio and

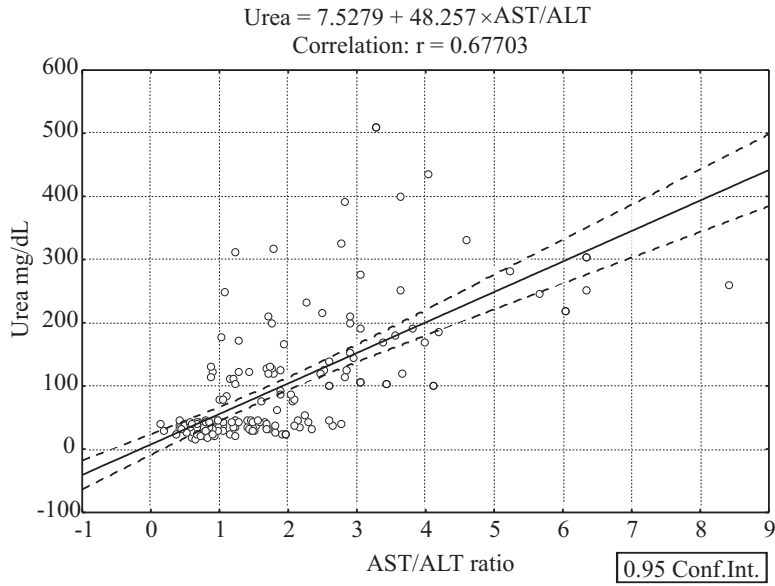

Fig. 3. Correlation between AST/ALT ratio and serum urea concentration in all 182 examined serum samples from dogs infected with $B$. canis (circles, particular cases; solid line, graph of the correlation function; dashed lines, $95 \%$ confidence interval; r, Pearson's correlation coefficient).

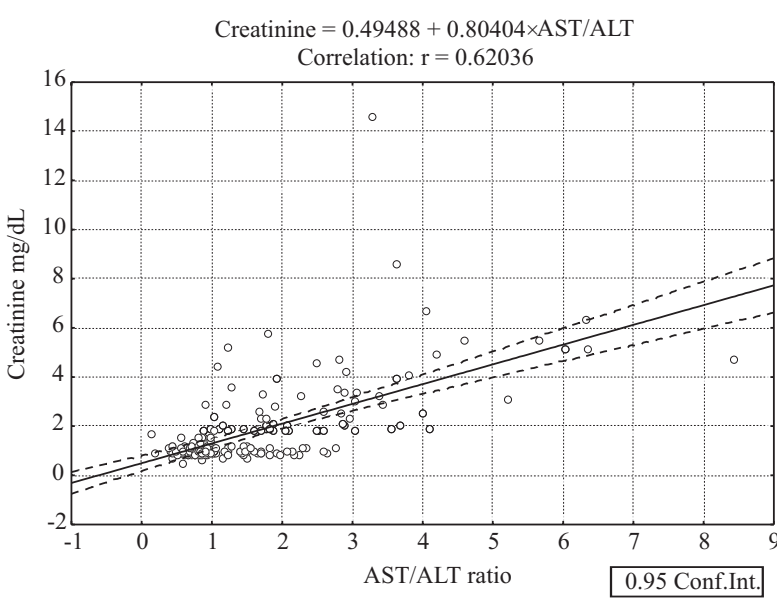

Fig. 4. Correlation between AST/ALT ratio and serum creatinine concentration in all 182 examined serum samples from dogs infected with B. canis (circles, particular cases; solid line, graph of the correlation function; dashed lines, 95\% confidence interval; r, Pearson's correlation coefficient).

creatinine concentration $(r=0.62, p=0.00)$ were observed. These correlations are presented in Figs. 3 and 4.

\section{Discussion}

The prevalence and severity of anaemia and azotaemia found in this work were similar to those reported in the previous studies on canine babesiosis from Poland (Zygner et al. 2007a,b). The more frequent increase in AST activity compared to ALT activity resulted in increased AST/ALT ratios. 
This study revealed that anaemia was not connected with increased AST/ALT ratio. Thus, it seems probable that injured erythrocytes did not significantly contribute to the increased AST activity observed in this work. Comparison of AST/ALT ratios in groups of azotaemic and non-azotaemic dogs showed that azotaemia was connected with a higher AST/ALT ratio. This finding suggested that kidney injury contributed to increased AST activity in these dogs. Moreover, high positive correlations between AST/ALT ratio, and serum urea and creatinine levels support this supposition. Increased AST activity observed in canine babesiosis may also be a result of rhabdomyolysis, however, this is a very rare complication of canine babesiosis (Jacobson 2006).

Kidney injury is one of the most common complications of canine babesiosis caused by B. canis (Máthé et al. 2006). Determination of serum urea and creatinine are the most commonly used renal screening tests in veterinary clinical practice. However, a previous study by De Scally et al. (2004) showed that an increase in serum urea and creatinine levels in canine babesiosis may be caused by non-renal factors. The results of the present study suggest that the AST/ALT ratio may be considered as a useful tool in the diagnosis of kidney injury during canine babesiosis. Increased AST/ALT ratio concurrent with an increase in serum urea and creatinine levels may be indicative of kidney injury. Moreover, this parameter can be used in combination with serum urea/creatinine ratio, which is a useful tool applied to the differentiation of pre-renal and renal azotaemia in humans (Jurado and Mattix 1998). Yet, further study is needed to determine reference intervals for the AST/ALT ratio in dogs and the cut off value of this parameter which would be indicative of kidney injury.

\section{References}

Abramowicz B (2008) Contribution of haemolysis to kidney and liver disturbances in dogs with babesiosis. Med Weter 64: 213-218.
Adaszek L, Winiarczyk S (2008) Molecular characterization of Babesia canis canis isolates from naturally infected dogs in Poland. Vet Parasitol 152: 235-241.

De Scally MP, Lobetti RG, Reyers F, Humphris D (2004) Are urea and creatinine values reliable indicators of azotaemia in canine babesiosis? J S Afr Vet Assoc 75: 121-124.

Furlanello T, Fiorio F, Caldin M, Lubas G, Solano-Gallego L (2005) Clinicopathological findings in naturally occurring cases of babesiosis caused by large form Babesia from dogs of northeastern Italy. Vet Parasitol 134: 77-85.

Irwin PJ (2009) Canine babesiosis: from molecular taxonomy to control. Parasit Vectors 2: S4.

Jacobson LS (2006) The South African form of severe and complicated canine babesiosis: Clinical advances 1994-2004. Vet Parasitol 138: 126-139.

Jurado R, Mattix H (1998) The decreased serum urea nitrogen-creatinine ratio. Arch Intern Med 158: 2509-2511 .

Kjemtrup AM, Wainwright K, Miller M, Penzhorn BL, Carreno RA (2006) Babesia conradae, sp. Nov., a small canine Babesia identified in California. Vet Parasitol 138: 103-111.

Máthé Á, Dobos-Kovács M, Vörös K (2007) Histological and ultrastructural studies of renal lesions in Babesia canis infected dogs treated with imidocarb. Acta Vet Hung 55: 511-523.

Máthé Á, Vörös K, Papp L, Reiczigel J (2006) Clinical manifestations of canine babesiosis in Hungary (63 cases). Acta Vet Hung 54: 367-385.

Welc-Falęciak R, Rodo A, Siński E, Bajer A (2009) Babesia canis and other tick-borne infections in dogs in Central Poland. Vet Parasitol 166: 191-198.

Zygner W, Gójska-Zygner O, Długosz E, Wędrychowicz $\mathrm{H}$ (2011) Liver enzyme activity in dogs infected with Babesia canis. Bull Vet Inst Pulawy 55: 423-427.

Zygner W, Gójska O, Rapacka G, Jaros D, Wędrychowicz $\mathrm{H}$ (2007a) Hematological changes during the course of canine babesiosis caused by large Babesia in domestic dogs in Warsaw (Poland). Vet Parasitol 145: 146-151.

Zygner W, Górski P, Wędrychowicz H (2009) Detection of the DNA of Borrelia afzelii, Anaplasma phagocytophilum and Babesia canis in blood samples from dogs in Warsaw. Vet Rec 164: 465-467.

Zygner W, Jaros S, Wędrychowicz H (2008) Prevalence of Babesia canis, Borrelia afzelii, and Anaplasma phagocytophilum infection in hard ticks removed from dogs in Warsaw (central Poland). Vet Parasitol 153: 139-142.

Zygner W, Rapacka G, Gójska-Zygner O, Długosz E, Wędrychowicz H (2007b) Biochemical abnormalities observed in serum of dogs infected with large Babesia in Warsaw (Poland). Pol J Vet Sci 10: 245-253. 